Article

\title{
Extended SSH Model in Non-Hermitian Waveguides with Alternating Real and Imaginary Couplings
}

\author{
Ziwei Fu ${ }^{1}$, Nianzu Fu ${ }^{1}$, Huaiyuan Zhang ${ }^{1}$, Zhe Wang ${ }^{1}$, Dong Zhao ${ }^{2, *}$ and Shaolin Ke ${ }^{1, *}$ \\ 1 Hubei Key Laboratory of Optical Information and Pattern Recognition, Wuhan Institute of Technology, \\ Wuhan 430205, China; fuziwei_wit@163.com (Z.F.); zzmrf1999@163.com (N.F.); witzhy@163.com (H.Z.); \\ wangzhe_wit@163.com (Z.W.) \\ 2 School of Electronics Information and Engineering, Hubei University of Science and Technology, \\ Xianning 437100, China \\ * Correspondence: zhaodong@hbust.edu.cn (D.Z.); keshaolin@wit.edu.cn (S.K.)
}

Received: 14 April 2020; Accepted: 13 May 2020; Published: 15 May 2020

\begin{abstract}
We studied the topological properties of an extended Su-Schrieffer-Heeger (SSH) model composed of a binary waveguide array with alternating real and imaginary couplings. The topological invariant of the periodic structures remained quantized with chiral symmetry even though the system was non-Hermitian. The numerical results indicated that phase transition arose when the absolute values of the two couplings were equal. The system supported a topological zero mode at the boundary of nontrivial structures when chiral symmetry was preserved. By adding onsite gain and loss to break chiral symmetry, the topological modes dominated in all supermodes with maximum absolute value of imaginary energy. This study enriches research on the SSH model in non-Hermitian systems and may find applications in optical routers and switches.
\end{abstract}

Keywords: topological phase; waveguides; non-Hermitian optics

\section{Introduction}

The Su-Schrieffer-Heeger (SSH) model is an important and basic model in describing band topology in condensed matter physics. It describes spinless fermions hopping on a one-dimensional lattice with staggered hopping amplitudes and provides intuitive ways to understand topological phenomenon such as topological invariant, phase transition, and bulk-boundary correspondence $[1,2]$. Recently, the SSH model has been widely investigated in various photonic systems such as optical waveguides [3-6] and nano particles [7]. The applications of topology in photonic systems are of great importance and offer new possibilities to design novel optical devices [8-12]. The photonic systems also provide a flexible platform for the SSH model, as the hopping of adjacent lattices can be controlled as desired by tuning distance via nanofabrication. Numerous extended models have been proposed to reveal further topological properties of the SSH model [13-19]. Anomalous $\pi$ modes are observed in coupled microwave waveguides by periodically modulating coupling strength [17]. Multiple topologically protected, bound states are theoretically indicated if the unit cell of the arrays is composed of multiple lattices [16]. The two-dimensional SSH model is able to retain nontrivial topological phase in the absence of Berry curvature and further can support second-order topological bound modes at its corners [18,19].

Another important extension is to examine the SSH model in non-Hermitian systems [20-23]. In contrast to Hermitian systems, the eigen energies may not be real, and the eigenstates become nonorthogonal [24-28]. Both eigen energies and eigenstates coalesce at the singularity of a non-Hermitian system, known as the exceptional point [24]. Interesting phenomena are found at the exceptional point such as giant Goos-Hänchen shifts [29], topological mode switching [30-33], 
and strong absorption [34]. In non-Hermitian systems, the topological properties are proven to be closely related to exceptional points and exhibit exceptional physics that has not manifested in Hermitian systems [20]. Non-Hermiticity can be introduced by adding onsite gain and loss at different lattices or by utilizing non-Hermitian hopping. Many fascinating and unusual topological phenomena are uncovered in the non-Hermitian SSH model. For example, all states, including bulk and edge sates, tend to locate at the boundary of the system, which is known as "skin effect" [20]. Topological lasing is experimentally realized in the systems with particle-hole symmetry [35]. The topological modes become extended in the whole system when the imaginary gauge potential is introduced [36]. When hopping is transformed into imaginary values, the SSH model is anti-parity-time-symmetric, and the topological phase transition would associate with the imaginary band structures rather than its real part [37].

In this work, we studied another extension of the non-Hermitian SSH model with alternating real and imaginary couplings. Imaginary coupling is accomplished by indirectly interacting two waveguides via a damped waveguide, which is known as adiabatic elimination. The technique was recently employed to realize both negative and imaginary couplings [38,39], and subsequently interesting phenomena are revealed such as synchronized oscillation [35] and extremely localized states in an Aharonov-Bohm cage [40]. Here, we use coupled mode theory to analyze the band structure and calculate its topological invariant in binary arrays. Furthermore, we also present full wave simulation by using planar optical waveguides to carry out the extended SSH model and numerically investigate the topological bound modes. The topological aspects with and without chiral symmetry are discussed in detail.

\section{Geometry and Theoretical Model}

The general SSH model depicts topological phenomenon in a one-dimensional dimer chain. Each unit cell has two lattices. Hopping between neighboring lattices in different unit cells is, $c_{1}$ and hopping between adjacent lattices in the same unit cell is $c_{2}$, which are real numbers. The SSH model has two energy bands under periodic boundary conditions, which degenerate at the Dirac point and experience band inversion as $c_{1}=c_{2}$. According to topological theory, this critical point is the topological phase transition point. The topological invariant of the SSH model can be characterized by the Berry phase $\varphi_{B}$, which is $\varphi_{B}=0$ for $c_{1}<c_{2}$ and for $\varphi_{B}=\pi$ for $c_{1}>c_{2}$. The system is topologically nontrivial for nonzero Berry phase and is able to sustain robust edge stats with zero energy at its boundary for open boundary conditions. In this work, we extended SSH by changing the intra-cell hopping to be imaginary, while the inter-cell hopping remained unchanged. Thus, the system becomes non-Hermitian, and the two hopping values are denoted $i c_{2}$ and $c_{1}$, respectively.

Hopping, which is also called coupling in optics, typically uses real numbers in weakly coupled waveguides. We used adiabatic elimination to modify the coupling to be imaginary. Adiabatic elimination is an efficient method to control coupling, and it requires two lattices indirectly coupled via an intermediate decoupled lattice. Details about the procedure of adiabatic elimination can be found in [41]. As shown in Figure 1a, we integrated an extra lattice $C$ into the bare lattices A and $B$. The center lattice $C$ serves as intermediate site and has strongly detuned onsite energy (also called propagation constants in waveguide systems). We set the imaginary part of onsite energies to be

$$
\delta_{\mathrm{A}, \mathrm{B}}=-i c_{2}, \delta_{\mathrm{C}}=i J^{2} / c_{2}
$$

where $J$ denotes the original coupling strength between lattice $C$ and A (B). The coupling coefficient $J$ is assumed to be a real number, as the dielectric waveguides are utilized in the following study. When detuning $\delta_{\mathrm{c}}$ is much larger than coupling $J$, then site $C$ can be adiabatically eliminated. Effective coupling between two sublattices A and B is determined to be $i c_{2}$. Consequently, imaginary hoping is employed through adiabatic elimination and appropriately fixes the onsite gain and loss in different lattices. More details about this technique can be found in recent studies [33]. Considering the 
condition of adiabatic elimination $\left(\delta_{\mathrm{c}}>>J\right)$, the original coupling $J$ should remarkably exceed the effective coupling strength $\left|i c_{2}\right|$. Figure $1 \mathrm{~b}$ shows the effective binary chain corresponding to Figure $1 \mathrm{a}$ after adiabatic elimination. The coupling in the whole system is alternatively real and imaginary, that is,$\ldots . .$. Im-Re-Im-Re-Im-Re ... ...

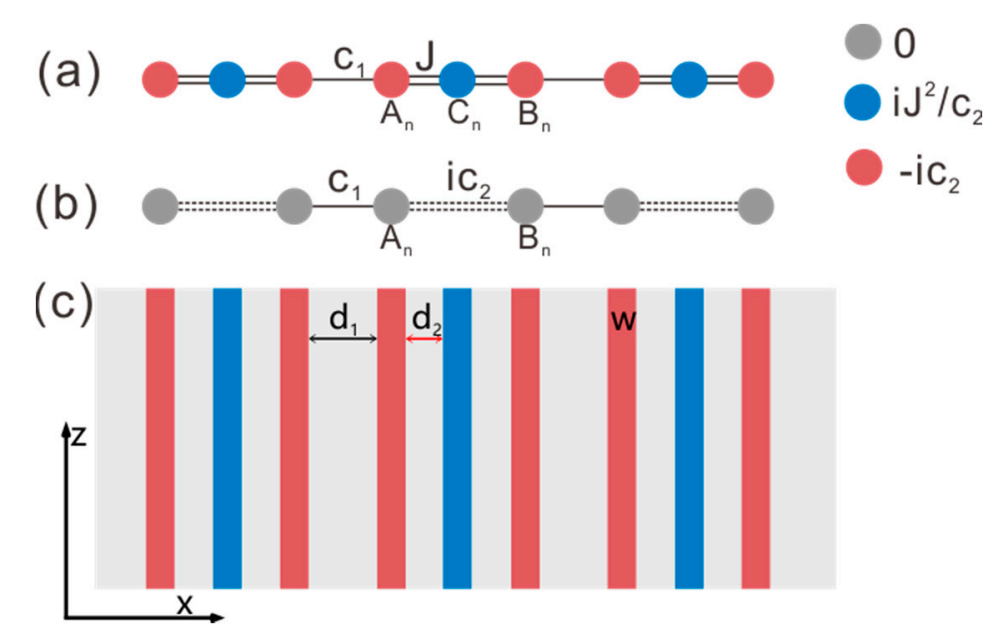

Figure 1. The geometry of the proposed Su-Schrieffer-Heeger (SSH) chain. (a) The suggested chain with assistant lattice. (b) The effective chain after adiabatic elimination. (c) The geometry of proposed planar waveguide.

We now investigate the band structure and the topological invariant in this extended SSH model. For the periodic boundary condition we can apply the Bloch theorem, and the Hamiltonian system is derived as

$$
\mathbf{H}=\left(\begin{array}{cc}
0 & i c_{2}+c_{1} e^{i \varphi} \\
i c_{2}+c_{1} e^{-i \varphi} & 0
\end{array}\right)
$$

where $\varphi$ denotes the Bloch momentum. Then, the eigenvalues are calculated to be

$$
\lambda_{ \pm}= \pm \sqrt{c_{1}^{2}-c_{2}^{2}+2 i c_{1} c_{2} \cos \varphi}
$$

The system has chiral symmetry, which is defined by $\sigma_{z} \mathbf{H}(\varphi) \sigma_{z}=-\mathbf{H}(\varphi)$, with $\sigma_{z}$ representing the Pauli matrix. The eigenvalues of the system come in \pm pairs due to chiral symmetry. In Equation (3), the eigenvalues appear in the form of \pm pair of square roots. Exceptional points emerge as two eigenvalues are of equal values, corresponding to the zero value of the square root. The condition takes place as $c_{1}=c_{2}$ at $\varphi= \pm \pi / 2$. This is different from the original SSH model and PT-symmetric SSH model, where the band touch points take place at the boundary of the Brillion zone as $\varphi=\pi$. At the exceptional points, the two eigenvalues are zero, and the eigenstates will coalesce. Apart from these points, the eigenvalues are complex. There is no special condition for real nor imaginary eigenvalues. This is also in contrast to PT symmetric systems.

The topology invariant of the system is generally characterized by the Berry phase. The Berry phase for individual bands is identified by

$$
\varphi_{\mathrm{B}}^{ \pm}=\oint_{k} i\left\langle\mu_{ \pm}|d / d k| \lambda_{ \pm}\right\rangle d k,
$$

where \pm marks two Bloch bands, and $k$ represents Bloch wavevector. $|\lambda\rangle$ and $\langle\mu|$ are the right and left eigenvectors of Hamiltonian $\mathrm{H}$, respectively. We numerically calculated the Berry phase, and the result is presented in Figure 2. As imaginary coupling was introduced, we obtained $\mathrm{H} \neq \mathrm{H}^{+}$, and the system was non-Hermitian. However, the Berry phase of two individual bands remained quantized, 
which was zero for $c_{2}>c_{1}$ and $\pi$ for $c_{2}<c_{1}$. The quantized Berry phase aids in chiral symmetry [42]. Chiral symmetry is the result of vanished onsite energy, which is broken with non-zero onsite energy. The condition of the topological phase transition is similar to that in the general SSH model and PT-symmetric systems.

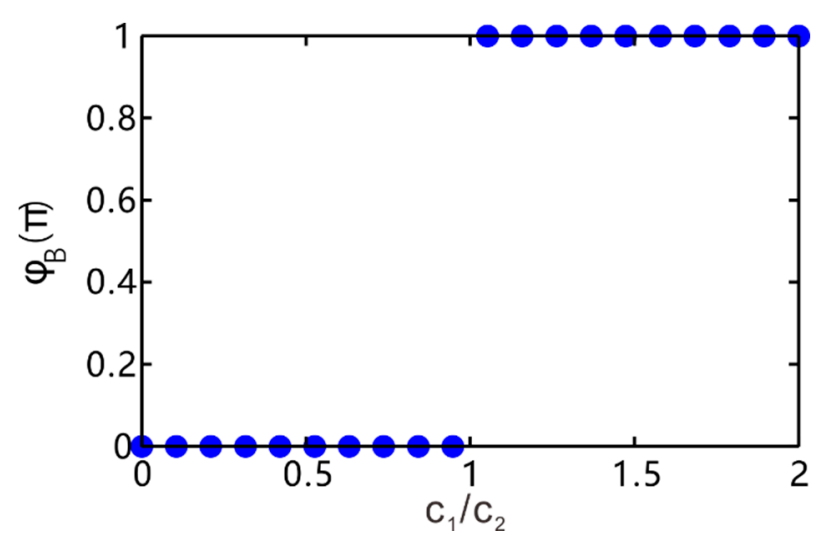

Figure 2. The Berry phase as a function of the ratio of relative coupling strength. The Berry phase of two band structures is equal, which is 0 as $c_{2}>c_{1}$ and $\pi$ as $c_{2}<c_{1}$.

\section{Bloch Mode}

We then used the coupled dielectric waveguide to carry out the above theorical proposal. The main purpose was to work out the parameters desired in adiabatic elimination. As discussed above, the loss added on the mediate waveguide should be extremely large. In practice, the effective system behaved well as $J>8 c_{2}$, and the loss should be $\delta_{2}>64 c_{2}$.

We discuss the planar waveguide arrays as illustrated in Figure 1c. The waveguide is periodic in the $x$ direction, and the light propagated in the $z$ direction. Every unit comprised three waveguides. Red denotes amplified bare waveguides, and blue denotes the mediate waveguides with loss. The real part of dielectric permittivity of each waveguide is fixed at $\varepsilon_{0}=12.25$, and the background dielectric is air with $\varepsilon_{\mathrm{d}}=1$. The wavelength of light is set at $\lambda=1.55 \mu \mathrm{m}$. The width of all waveguides is constant and fixed at $w=0.24 \mu \mathrm{m}$. Transverse magnetic polarization is accounted for in this study, and each individual waveguide is determined to sustain a single mode with propagation constant $\beta_{0}=7.7617 \mu \mathrm{m}^{-1}$. This can be regarded as the background onsite energy in the Hamiltonian $\mathrm{H}$ and will not violate the conclusions. The spatial spacing between bare and assistant waveguides is fixed at $d_{2}=0.5 \mu \mathrm{m}$. The nearest coupling $J$ between different waveguides can be extracted from a double-waveguide coupler. Then, we can use Equation (1) to find out the detuned propagation constants for certain imaginary coupling $c_{2}$. The detuned dielectric permittivity for bare and assistant waveguides can be determined by simulating the propagation constants in a single waveguide. For instance, if the effective imaginary coupling was chosen as $c_{2}=4 \times 10^{-3} \mu \mathrm{m}^{-1}$, the imaginary parts of dielectric permittivity in waveguide $A(B)$ and $C$ should be $-0.0056 \mathrm{i}$ and $0.7769 \mathrm{i}$, respectively. The spacing $d_{1}$ will be adjusted to satisfy different real coupling strengths. More detail about how to determine the related parameters can be found in our recent studies $[25,37]$.

We use full wave simulation to calculate the band structures. Figure 3 shows the band structures in the first Brillion zone for periodic boundary condition for several coupling strengths. In all cases, the effective imaginary coupling is fixed at $c_{2}=4 \times 10^{-3} \mu \mathrm{m}^{-1}$. The interlayer coupling $c_{1}$ is modulated by modifying the special spacing $d_{1}$. There are two energy bands (depicted by red and blue colors) in the structure, corresponding to two different Bloch modes. We define two different modes according to the continuity of complex eigenvalues. For example, in Figure 3 e, the real parts of two eigenvalues are always separated and can represent two modes. Then, we plot the corresponding imaginary parts in Figure 3f. The imaginary part may cross each other, but they can be distinguished by their real part and the colours can be determined. The two bands come in pairs with $\pm\left(\beta-\beta_{0}\right)$ as a result of 
chiral symmetry. Both energy bands are complex for all different couplings. This is different from a PT symmetric system where the energy bands can be purely real for special conditions. In Figure $3 a, b$, the relative coupling strength is $c_{1}<c_{2}$. In this case, the real part of two energy bands is crossed and the imaginary part is separated. The cross points always arise in the condition of $\varphi= \pm \pi / 2$. In Figure $3 c, d$, the absolute values of intra- and inter-layer couplings are identical. This is the phase transition point. In this case, both the real and imaginary parts of two energy bands coalesce simultaneously at $\varphi= \pm \pi / 2$ with $\beta-\beta_{0}=0$. The results are accordant with the prediction by the coupled mode theory. In Figure 3e,f, the relative coupling strength satisfies $c_{1}>c_{2}$. In this condition, the real part of two energy bands is separated, and the imaginary parts of two energy bands cross each other. The cross points always emerge when the Bloch momentum is $\varphi= \pm \pi / 2$. This can be understood form Equation (1) as the term $2 i c_{1} c_{2} \cos \varphi$ vanishes at these two points.
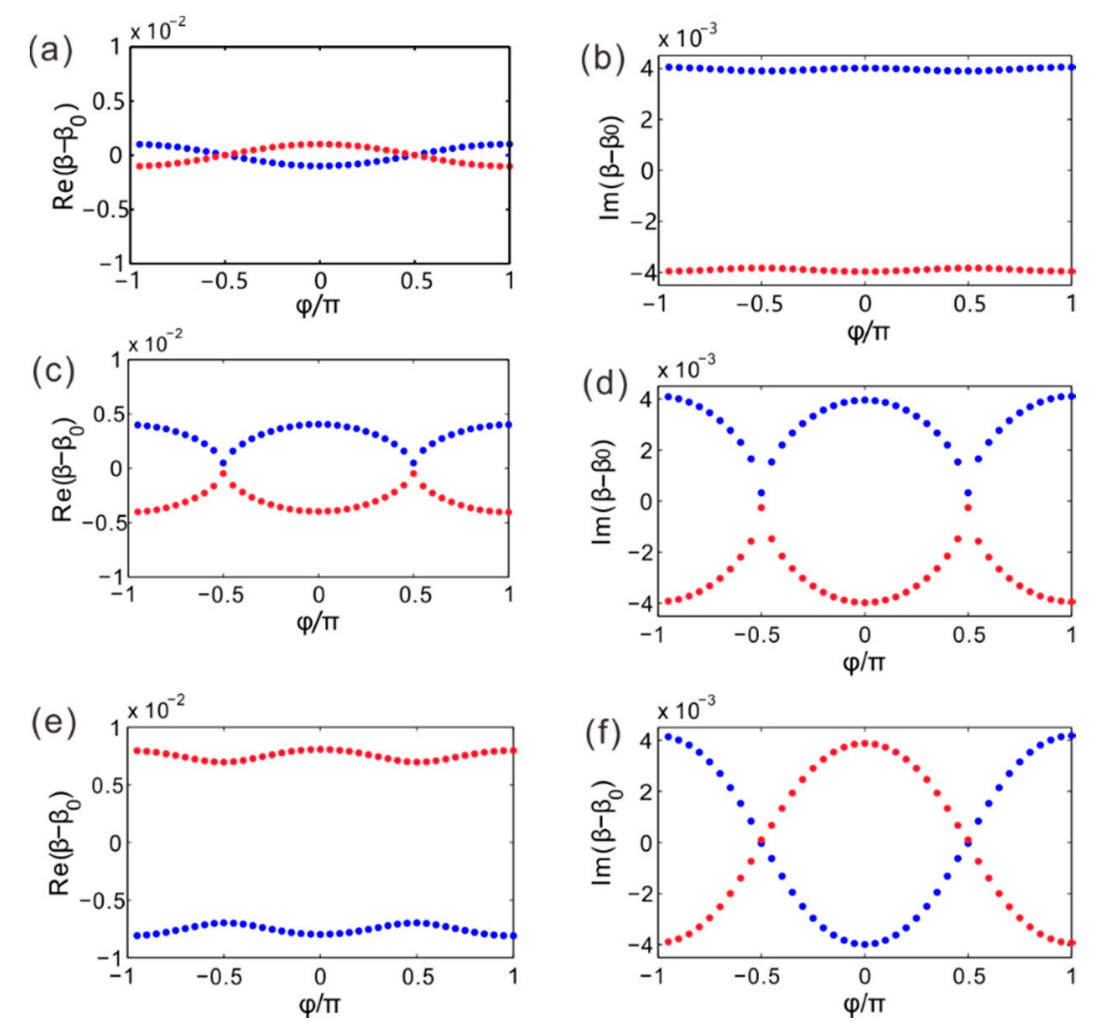

Figure 3. The numerical band structures of periodic waveguide arrays for different coupling strengths. (a) and (b) are the real part and imaginary part as $c_{1}=1 \times 10^{-3} \mu \mathrm{m}^{-1}$ and $c_{2}=4 \times 10^{-3} \mu \mathrm{m}^{-1}$ (c) and (d) are the real part and imaginary part as $c_{1}=c_{2}=4 \times 10^{-3} \mu \mathrm{m}^{-1}$. (e) and (f) are the real part and imaginary part as $c_{1}=8 \times 10^{-3} \mu^{-1}$ and $c_{2}=4 \times 10^{-3} \mu \mathrm{m}^{-1}$.

\section{Topological Edge Modes}

We then analyzed the optical modes in the waveguides with open boundary conditions. The most prominent feature was that the edge modes in the array had no vanished topological invariants, which is known as bulk-edge correspondence.

In Figure 4, we plot the propagation constants of all supermodes for topological trivial and nontrivial structures. Figure $4 \mathrm{a}, \mathrm{b}$ exhibits the real and imaginary parts of propagation constants as $c_{1}=8 \times 10^{-3} \mu^{-1}$ and $c_{2}=4 \times 10^{-3} \mu \mathrm{m}^{-1}$, respectively. In this case, the structure is topological nontrivial and can support topological edge modes. One can see the real part of propagation constants has an energy gap, and two zero modes are in the gap. The propagation constants of these two modes are equal to that in a single waveguide and can be regarded as zero modes. Figure $4 \mathrm{c}, \mathrm{d}$ shows the real and imaginary parts of propagation constants as $c_{1}=1 \times 10^{-3} \mu \mathrm{m}^{-1}$ and $c_{2}=4 \times 10^{-3} \mu \mathrm{m}^{-1}$, 
respectively. The Berry phase vanishes, and the structure will not allow for topological edge modes. We cannot see zero mode in the gap of spectra of propagation constants.
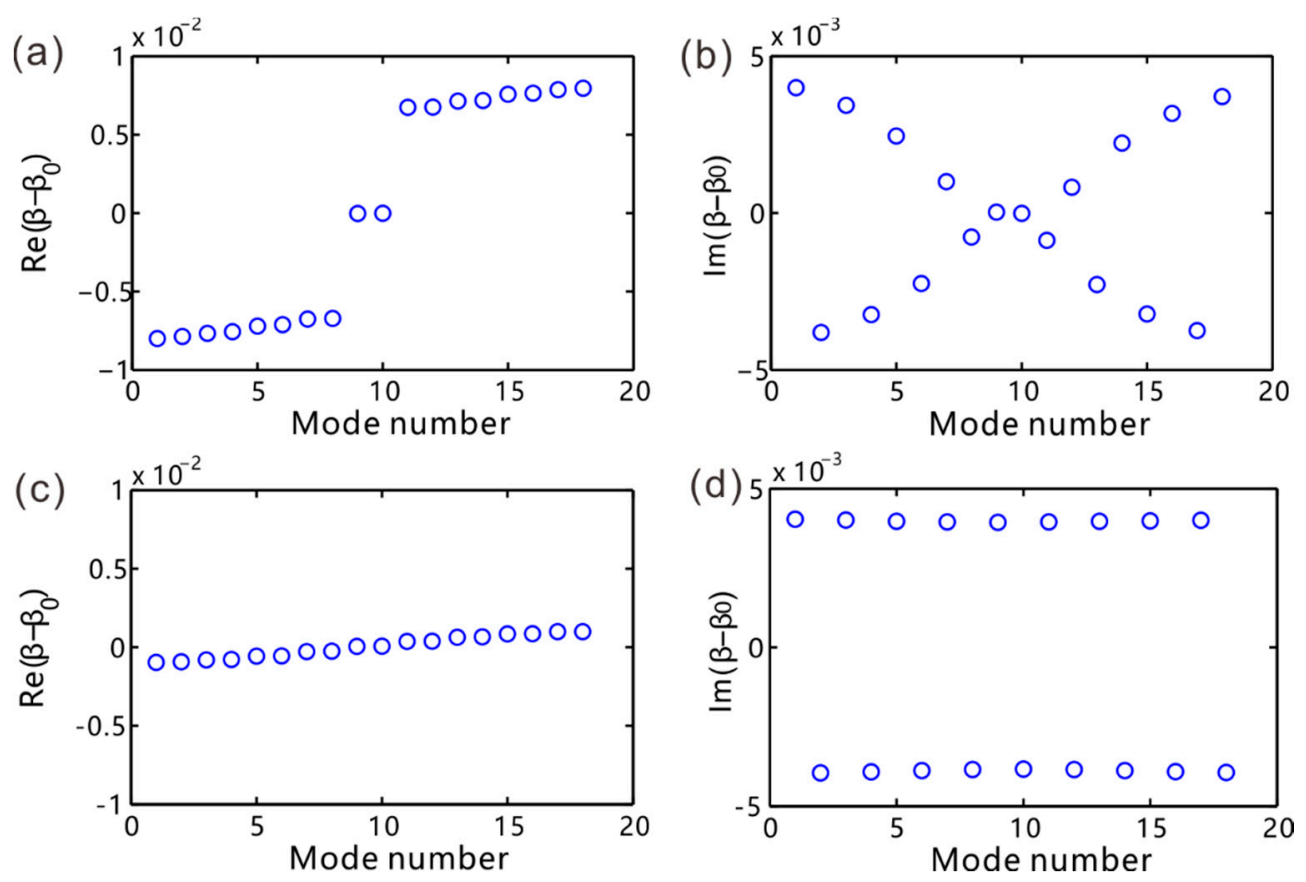

Figure 4. The propagation constants in finite waveguide arrays for two different coupling strengths. (a) and (b) are the real and imaginary parts of propagation constants as $c_{1}>c_{2}$. (c) and (d) are the real and imaginary parts of propagation constants as $c_{1}<c_{2}$.

Figure 5 illustrates the field distributions of two topological edge modes. In Figure 5a,b, we plot the distributions of electric field norm $|\mathbf{E}|$ and component $E_{x}$ for one topological edge mode. The values of norm of the electric field are positive, while the component of the electric field can be positive and negative. Therefore, we used different colormaps. In Figure $5 \mathrm{a}$, the dark color represents zero valued field, and red color stands for the strong field. The energy is confined at two terminations of the structure and exponentially decreases away from the terminations. The energy at two ends are almost equal. In Figure $5 b$, the blue color denotes the negative values, and the red stands for positive values. One can see this mode is anti-symmetric with respect to the center axis of the structure. Figure $5 c, d$ shows the distributions of electric field norm $|\mathbf{E}|$ and component $E_{x}$ for the other edge mode. The norm of the field is also distributed at two terminations of the structure. One can see this mode is symmetric from the distributions of $E_{x}$.

The above discussed the extended SSH model has chiral symmetry, which can be broken by adding onsite energy. In waveguides, the onsite energy is propagation constants, which can be altered by real and imaginary parts of permittivity. Next, we considered the case by tuning the imaginary part of propagation constants. In each unit cell, the two bare waveguides have alternating detuned propagation constants $-i \delta / 2$ and $i \delta / 2$. Other parameters remain unchanged from that used in Figure $4 a$.

Figure $6 \mathrm{a}$ plots the propagation constants in complex plane as $\delta=0$. Blue dots denote the bulk modes, and the red dots represent the edge mode. The two edge modes are of zero energy. Figure $6 \mathrm{~b}$ shows the propagation constants of all supermodes for $\delta=0.015$. The imaginary part of propagation constants of edge modes now has the largest absolute value, while the real part of propagation constants remains zero. The mode with positive imaginary propagation constants is decayed, while the mode with negative imaginary is amplified. Therefore, one of the edge modes will dominate after suitable evolution, which is important in many applications. In Figure $6 c$, we plot $\operatorname{Im}(\beta)$ of the edge mode and the largest value of imaginary propagation constant of bulk mode as a function of detuning $\delta$. The imaginary part of the edge mode linearly increases with the increase of detuning $\delta$ 
according to $\operatorname{Im}(\beta)=\delta / 2$. In contrast, the $\operatorname{Im}(\beta)$ of bulk modes displays a quite slow increase. As a result, the imaginary propagation constants of edge modes exceed that of bulk modes by choosing a proper $\delta$. As $\delta$ further increases, the propagation constants of bulk modes and edge modes tend to be equal. This can be understood from the coupled mode equation. According to the coupled mode equation, the eigenvalues of bulk modes are derived as

$$
\lambda_{ \pm}= \pm \sqrt{-\frac{\delta^{2}}{4}+c_{1}^{2}-c_{2}^{2}+2 i c_{1} c_{2} \cos \varphi}
$$

(a)

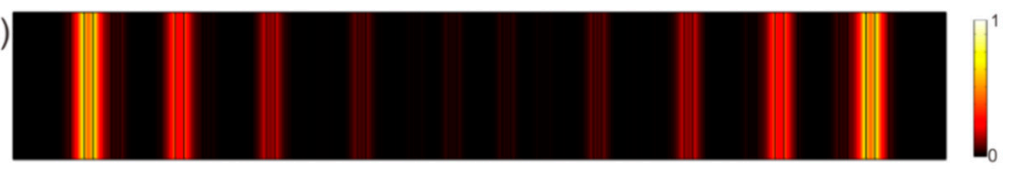

(b)

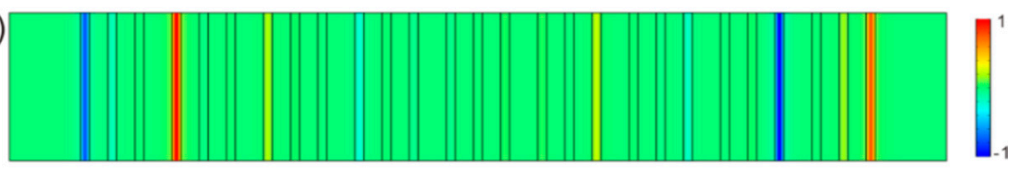

(c)

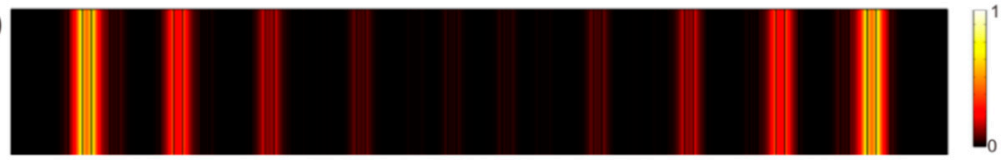

(d)

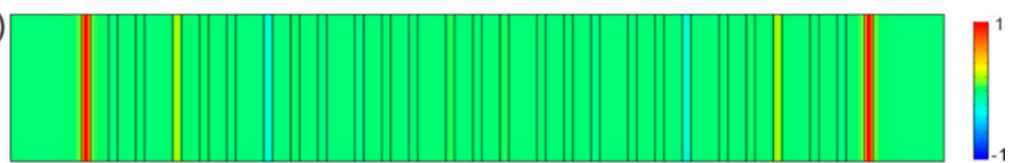

Figure 5. The field distributions of two edge modes. (a) and (c) are the norm of electric field (|E|) for two topological edge modes. (b) and (d) are the component of electric field along the $x$ direction $\left(E_{x}\right)$.
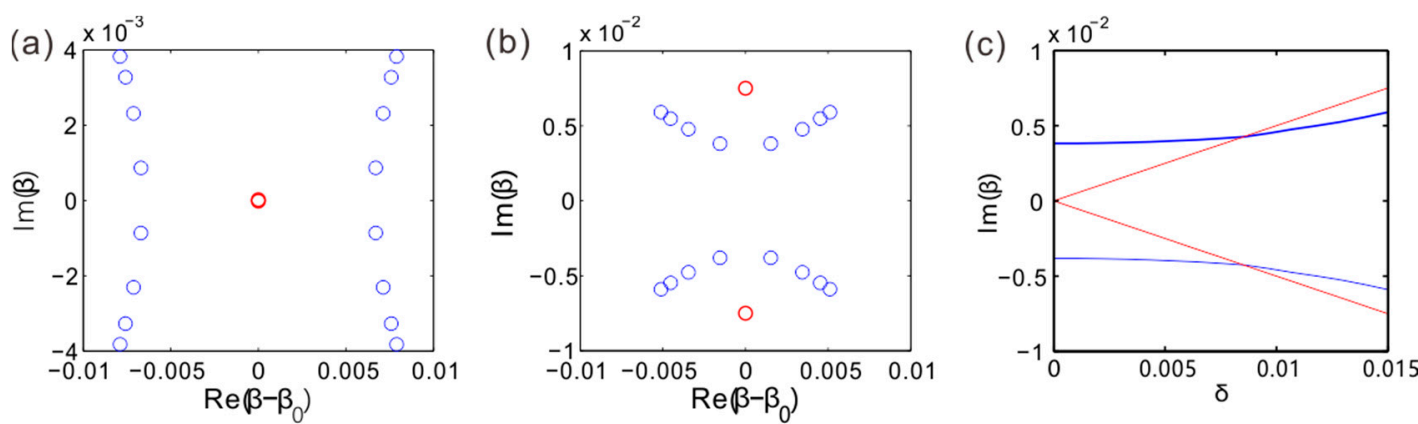

(d)

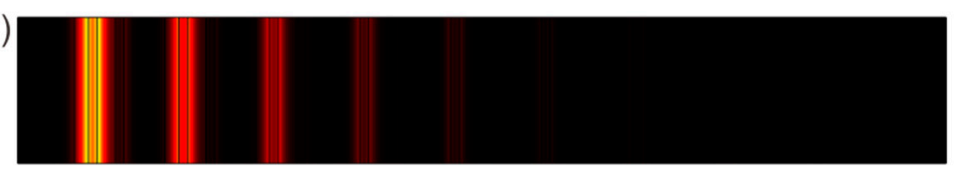

(e)

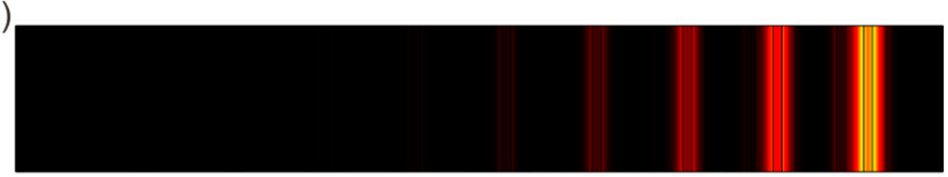

Figure 6. The supermodes for different detuned propagation constantans $i \delta$. (a) The supermode for $\delta=0$. (b) The supermode for $\delta=0.015$. (c) $\operatorname{Im}(\beta)$ as a function of $\delta$. The blue lines denote the maximum imaginary part of propagation constants for bulk mode. The red lines denote the topological edge mode. (d) and (e) are the field distributions ( $|\mathbf{E}|)$ of two edge modes for $\delta=0.015$. 
The eigenvalues of edge modes are $\beta= \pm i \delta / 2$. As $\delta>>c_{1}, c_{2}$, the eigenvalues of all bulk modes also tend to be $\beta= \pm i \delta / 2$. Therefore, the propagation constants of edge modes and bulk modes tend to be equal for extremely large $\delta$. We also illustrate the field distributions of two edge modes for $\delta=0.015$, as shown in Figure $6 \mathrm{~d}$,e. When chiral symmetry is broken, the fields of both edge modes are confined at one termination of the waveguides; one is at left and the other is right. Moreover, the fields of edge mode in Figure $6 \mathrm{~d}$ are only confined at the amplified waveguides, while the fields of the other edge mode (Figure 6e) are mainly concentrated on attenuated waveguides. As a result, the two edge modes experience the strongest gain or loss.

The proposed system may be experimentally realized using Fe-doped $\mathrm{LiNbO}_{3}$ waveguides [43]. In this system, the optical gain can be accomplished through two-wave mixing. By selectively pumping the waveguides, alternating gain and loss then will be achieved. Moreover, imaginary coupling based on adiabatic elimination has also recently been realized in $\mathrm{LiNbO} 3$ waveguides [39]. The huge loss is achieved by incorporated $\mathrm{Cr}$ into the assistant waveguide. Therefore, we think the most probable realistic scenario may be realized in $\mathrm{LiNbO}_{3}$ waveguides.

\section{Conclusions}

In summary, we have investigated an extended SSH model in optical waveguides with real and imaginary couplings. Imaginary coupling is employed through adiabatic elimination and appropriately fixing the onsite gain and loss in different waveguides. The system supports two bands, and the topological invariant of the system remains quantized even though the system is non-Hermitian. The energy bands of the periodic system are generally complex but coalesce at the exceptional point, with the Bloch momentum zero value of $\varphi= \pm \pi / 2$. We show the topological phase transition also takes place at this point. Topological zero mode is supported at the edge of nontrivial structures when chiral symmetry is preserved. By adding onsite gain and loss to break chiral symmetry, the topological bound modes no longer remain at zero energy and dominate in all supermodes with the maximum absolute value of the imaginary part of propagation constants. This work enriches study of the SSH model and may find great applications in optical routers and switches.

Author Contributions: Conceptualization, S.K.; software, Z.F. and N.F.; formal analysis, Z.F., Z.W. and H.Z.; writing-original draft preparation, D.Z.; writing—review and editing, S.K. All authors have read and agreed to the published version of the manuscript.

Funding: This research was funded by Program for Distinguished Middle-aged and Young Innovative Research Team in Higher Education of Hubei, China, grant number T201806; the Natural Science Foundation of Hubei Province, grant number 2017CFB150; and the 14th President's Fund of Wuhan Institute of Technology.

Conflicts of Interest: The authors declare no conflict of interest.

\section{References}

1. Su, W.P.; Schrieffer, J.R.; Heeger, A.J. Solitons in Polyacetylene. Phys. Rev. Lett. 1978, 42, 1698. [CrossRef]

2. Asbóth, J.K.; Oroszlány, L.; Pályi, A. The Su-Schrieffer-Heeger (SSH) Model. In A Short Course on Topological Insulators. Lecture Notes in Physics; Springer: Cham, Switzerland, 2016; p. 919.

3. Blanco-Redondo, A.; Andrea, I.; Collins, M.; Harari, G.; Lumer, Y.; Rechtsman, M.; Eggleton, B.; Segev, M. Topological optical waveguiding in silicon and the transition between topological and trivial defect states. Phys. Rev. Lett. 2016, 116, 163901. [CrossRef]

4. Cheng, Q.; Pan, Y.; Wang, Q.; Li, T.; Zhu, S. Topologically protected interface mode in plasmonic waveguide arrays. Laser Photon. Rev. 2015, 9, 392-398. [CrossRef]

5. Ge, L.; Wang, L.; Xiao, M.; Wen, W.; Chan, C.; Han, D. Topological edge modes in multilayer graphene systems. Opt. Express 2015, 23, 21585-21595. [CrossRef] [PubMed]

6. Ke, S.; Wang, B.; Long, H.; Wang, K.; Lu, P. Topological edge modes in non-Hermitian plasmonic waveguide arrays. Opt. Express 2017, 25, 11132-11143. [CrossRef]

7. Wang, B.X.; Zhao, C.Y. Wideband tunable infrared topological plasmon polaritons in dimerized chains of doped-silicon nanoparticles. J. Appl. Phys. 2020, 127, 073106. [CrossRef] 
8. Peng, Y.G.; Qin, C.; Zhao, D.; Shen, Y.; Xu, X.; Bao, M.; Jia, H.; Zhu, X. Experimental demonstration of anomalous Floquet topological insulator for sound. Nat. Commun. 2016, 7, 13368. [CrossRef]

9. Xu, S.; Zhou, Q.; Zhao, D.; Belic, M.; Zhao, Y. Spatiotemporal solitons in cold Rydberg atomic gases with Bessel optical lattices. Appl. Math. Lett. 2020, 106, 106230. [CrossRef]

10. Lu, L.; Joannopoulos, D.; Soljačić, M. Topological photonics. Nat. Photon. 2014, 8, 821-829. [CrossRef]

11. Deng, H.; Chen, X.; Panoiu, N.; Ye, F. Topological surface plasmons in superlattices with changing sign of the average permittivity. Opt. Lett. 2016, 41, 4281-4284. [CrossRef]

12. Xu, C.; Zhang, P.; Zhao, D.; Guo, H.; Huang, M.; Ke, S. Plasmonic Jackiw-Rebbi Modes in Graphene Waveguide Arrays. Appl. Sci. 2019, 9, 4152. [CrossRef]

13. Xie, D.; Gou, W.; Xiao, T.; Gadway, B.; Yan, B. Topological characterizations of an extended Su-Schrieffer-Heeger model. npj Quantum Inf. 2019, 5, 55. [CrossRef]

14. Fu, N.; Fu, Z.; Zhang, H.; Liao, Q.; Zhao, D.; Ke, S. Topological bound modes in optical waveguide arrays with alternating positive and negative couplings. Opt. Quantum Electron. 2020, 52, 61. [CrossRef]

15. Meng, P.; Zhao, D.; Zhong, D.; Liu, W. Topological plasmonic modes in graphene-coated nanowire arrays. Opt. Quantum Electron. 2019, 51, 156. [CrossRef]

16. Wang, F.; Ke, S.; Qin, C.; Wang, B.; Long, H.; Wang, K.; Lu, P. Topological interface modes in graphene multilayer arrays. Opt. Laser Technol. 2018, 103, 272-278. [CrossRef]

17. Cheng, Y.Q.; Pan, H.; Wang, C.; Zhang, D.; Yu, A.; Gover, H.; Zhang, T.; Li, L.Z.; Zhu, S. Observation of Anomalous $\pi$ Modes in Photonic Floquet Engineering. Phys. Rev. Lett. 2019, 122, 173901. [CrossRef]

18. Liu, F.; Wakabayashi, K. Novel Topological Phase with a Zero Berry Curvature. Phys. Rev. Lett. 2017, 118, 076803. [CrossRef]

19. Chen, X.; Deng, W.; Shi, F.; Zhao, F.; Chen, M.; Dong, J. Direct observation of corner states in second-order topological photonic crystal slabs. Phys. Rev. Lett. 2019, 122, 233902. [CrossRef]

20. Yao, S.; Wang, Z. Edge States and Topological Invariants of Non-Hermitian Systems. Phys. Rev. Lett. 2018, 121, 086803. [CrossRef]

21. Silveirinha, M.G. Topological theory of non-Hermitian photonic systems. Phys. Rev. B 2019, 99, 125155. [CrossRef]

22. Yuce, C. Topological phase in a non-Hermitian PT symmetric system. Phys. Lett. A 2015, 379, 1213. [CrossRef]

23. Yuce, C.; Ramezani, H. Topological states in a non-Hermitian two-dimensional Su-SchriefferHeeger model. Phys. Rev. A 2019, 100, 032102. [CrossRef]

24. Miri, M.; Alù, A. Exceptional points in optics and photonics. Science 2019, 363, 6422. [CrossRef]

25. Ke, S.; Zhao, D.; Liu, Q.; Wu, S.; Wang, B.; Lu, P. Optical imaginary directional couplers. J. Lightwave Technol. 2018, 36, 2510-2516. [CrossRef]

26. Zhao, D.; Xu, B.; Guo, H.; Xu, W.; Zhong, D.; Ke, S. Low Threshold Optical Bistability in Aperiodic PT-Symmetric Lattices Composited with Fibonacci Sequence Dielectrics and Graphene. Appl. Sci. 2019, 9, 5125. [CrossRef]

27. Zhao, D.; Ke, S.; Hu, Y.; Wang, B.; Lu, P. Optical bistability of graphene embedded in parity-time-symmetric photonic lattices. JOSA B 2019, 36, 1731-1737. [CrossRef]

28. Liu, F.; Zhao, D.; Cao, H.; Xu, B.; Xu, W.; Ke, S. Exceptional Points in Non-Hermitian Photonic Crystals Incorporated with a Defect. Appl. Sci. 2020, 10, 823. [CrossRef]

29. Zhao, D.; Zhong, D.; Hu, Y.; Ke, S.; Liu, W. Imaginary modulation inducing giant spatial Goos-Hänchen shifts in one-dimensional defective photonic lattices. Opt. Quantum Electron. 2019, 51, 113. [CrossRef]

30. Zhang, X.; Jiang, T.; Chan, C.T. Dynamically encircling an exceptional point in anti-parity time symmetric systems: Asymmetric mode switching for symmetry-broken modes. Light Sci. Appl. 2019, 8, 88. [CrossRef]

31. Ke, S.; Wang, B.; Qin, C.; Long, H.; Wang, K.; Lu, P. Exceptional points and asymmetric mode switching in plasmonic waveguides. J. Lightwave Technol. 2016, 34, 5258-5262. [CrossRef]

32. Ke, S.; Wang, B.; Long, H.; Wang, K.; Lu, P. Topological mode switching in a graphene doublet with exceptional points. Opt. Quantum Electron. 2017, 49, 1-12. [CrossRef]

33. Ke, S.; Zhao, D.; Liu, Q.; Liu, W. Adiabatic transfer of surface plasmons in non-Hermitian graphene waveguides. Opt. Quantum Electron. 2018, 50, 393. [CrossRef]

34. Ke, S.; Liu, J.; Liu, Q.; Zhao, D.; Liu, W. Strong absorption near exceptional points in plasmonic waveguide arrays. Opt. Quantum Electron. 2018, 50, 31. [CrossRef] 
35. Parto, M.; Wittek, S.; Hodaei, H.; Harari, G.; Miguel, A.B.; Ren, J.; Mikael, C.R.; Segev, M.; Demetrios, N.C.; Khajavikhan, M. Edge-Mode Lasing in 1D Topological Active Arrays. Phys. Rev. Lett. 2018, 120, 113901. [CrossRef]

36. Yuce, C. Non-Hermitian anomalous skin effect. Phys. Lett. A 2020, 384, 126094. [CrossRef]

37. Ke, S.; Zhao, D.; Liu, J.; Liu, Q.; Liao, Q.; Wang, B.; Lu, P. Topological bound modes in anti-PT symmetric optical waveguide arrays. Opt. Express 2019, 27, 13858-13870. [CrossRef]

38. Keil, R.; Poli, C.; Heinrich, M.; Arkinstall, J.; Weihs, G.; Schomerus, H.; Szameit, A. Universal Sign Control of Coupling in Tight-Binding Lattices. Phys. Rev. Lett. 2016, 116, 213901. [CrossRef]

39. Fan, H.; Chen, J.; Zhao, Z.; Wen, J.; Huang, Y. Anti-parity-time Symmetry in Passive Nanophotonics. arXiv 2020, arXiv:2003.11151.

40. Kremer, M.; Petrides, I.; Meyer, E.; Heinrich, M.; Zilberberg, O.; Szameit, A. A square-root topological insulator with non-quantized indices realized with photonic Aharonov-Bohm cages. Nat. Commun. 2020, 11, 907. [CrossRef]

41. Yang, F.; Liu, Y.; You, L. Anti-PT symmetry in dissipatively coupled optical systems. Phys. Rev. B 2017, 96, 053845. [CrossRef]

42. Lieu, S. Topological phases in the non-Hermitian Su-Schrieffer-Heeger model. Phys. Rev. B 2018, 9, 045106. [CrossRef]

43. Rüter, C.; Makris, K.; El-Ganainy, R.; Christodoulides, D.; Segev, M.; Kip, D. Observation of parity-time symmetry in optics. Nat. Phys. 2010, 6, 192-195. [CrossRef]

(C) 2020 by the authors. Licensee MDPI, Basel, Switzerland. This article is an open access article distributed under the terms and conditions of the Creative Commons Attribution (CC BY) license (http://creativecommons.org/licenses/by/4.0/). 